\title{
Haldane Topological Orders in Motzkin Spin Chains
}

\author{
L. Barbiero, ${ }^{1,2}$ L. Dell'Anna, ${ }^{3}$ A. Trombettoni, ${ }^{1,4}$ and V. E. Korepin ${ }^{5}$ \\ ${ }^{1}$ CNR-IOM DEMOCRITOS Simulation Center, Via Bonomea 265, I-34136 Trieste, Italy \\ ${ }^{2}$ Center for Nonlinear Phenomena and Complex Systems, \\ Universit Libre de Bruxelles, CP 231, Campus Plaine, B-1050 Brussels, Belgium \\ ${ }^{3}$ Dipartimento di Fisica e Astronomia “G. Galilei”, Università di Padova, via F. Marzolo 8, I-35131, Padova, Italy \\ ${ }^{4}$ SISSA and INFN, Sezione di Trieste, Via Bonomea 265, I-34136 Trieste, Italy \\ ${ }^{5}$ C. N. Yang Institute for Theoretical Physics, Stony Brook University, NY 11794, USA
}

\begin{abstract}
Motzkin spin chains are frustration-free models whose ground-state is a combination of Motzkin paths. The weight of such path contributions can be controlled by a deformation parameter $t$. As a function of the latter these models, beside the formation of domain wall structures, exhibit gapped Haldane topological orders with constant decay of the string order parameters for $t<1$. A behavior compatible with a Berezinskii-KosterlitzThouless phase transition at $t=1$ is also presented. By means of numerical calculations we show that the topological properties of the Haldane phases depend on the spin value. This allows to classify different kinds of hidden antiferromagnetic Haldane gapped regimes associated to nontrivial features like symmetry-protected topological order. Our results from one side allow to clarify the physical properties of Motzkin frustration-free chains and from the other suggest them as a new interesting and paradigmatic class of local spin Hamiltonians.
\end{abstract}

PACS numbers:

Spin chains play a crucial role in many fundamental physical phenomena like magnetism [1], quantum phase transitions [2], topological orders [3] and quantum computation [4]. A fundamental contribute to the understanding of spin chains is provided by the the seminal papers by Haldane [5] where a new topological phase, the Haldane phase (HP), uniquely detectable via a non-local string order parameter (SOP) [6] has been discovered for spin-1 XXZ Heisenberg chains. This has driven significant efforts to look for new kinds of models whose topological order can be described in terms of a SOP [7] motivating the discovery of the celebrated AffleckKennedy-Lieb-Tasaki (AKLT) model [8]. Although the argument of Haldane is given for integer spin chains, only integer spin XXZ-like and AKLT-like chains own topological $\mathrm{HP}$ and is therefore non-trivial to find and study new classes of Hamiltonians where HP emerges. Thanks to the strongest quantum "resource", namely the entanglement, spin models have also a fundamental role in the simulation of quantum logical gates for quantum computation [4]. For this reason finding and studying Hamiltonians with highly entangled spins is currently one of the most challenging and intriguing fields in quantum physics.

In this direction local integer frustration-free spin Hamiltonians whose ground-state can be expressed as a combination of Motzkin paths [9] have been recently introduced [10, 11]. Among others interesting aspects, their importance is given by the fact that they own a level of entanglement entropy which strongly exceeds the one exhibited by other previously known local models. Relevantly, also for half-integer spins, a similar class of Hamiltonians, the Fredkin spin chains, exhibiting the same features [12, 13] has been introduced. In addition to their entanglement properties Motzkin chains own further very peculiar properties. Indeed, even if they are purely local models, for high spin values $s$ (i.e., $s \geq 2$ ) they behave as de facto long range Hamiltonians being able to violate cluster decomposition properties (CDP) and area law (AL) scaling

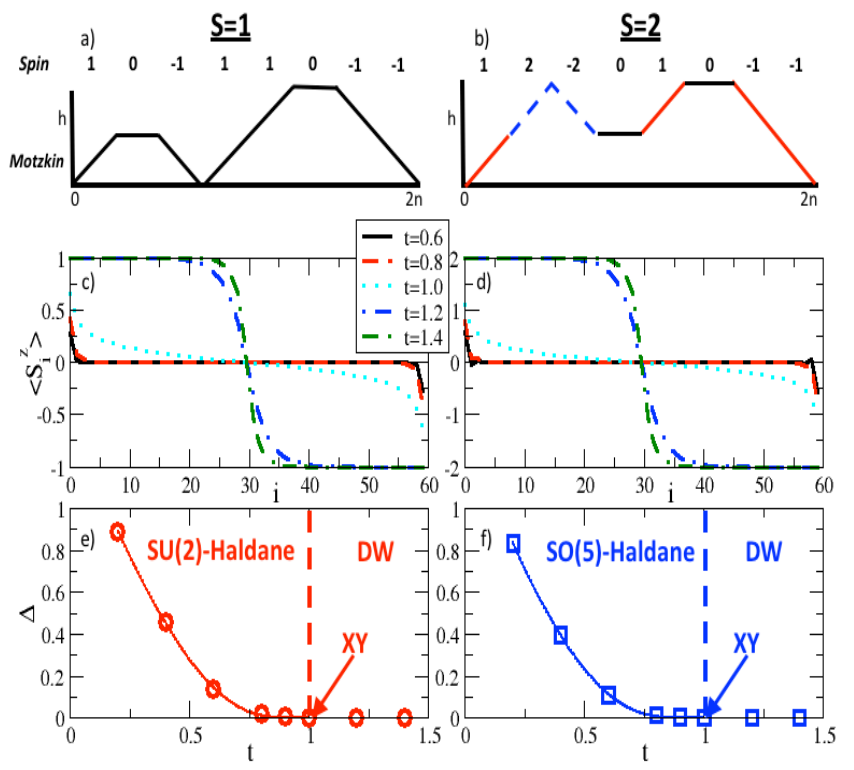

Figure 1: (Color online) Upper panels: Cartoons of a possible Motzkin path and its representation in terms of spins for the two cases $a$ ) uncolored $s=1$ and $b$ ) colored $s=2$. Central panels: DMRG local magnetization for a system of length $2 n=60$ at different $t$ deformation values $\left\langle S_{i}^{z}\right\rangle$ for $c$ ) $s=1$ and $d$ ) $s=2$. Lower panels: Thermodynamic limit of the gap $\Delta=E_{1}-E_{0}$ as a function of $t$ for $e$ ) $s=1$ (red circles) and $f$ ) $s=2$ (blue squares). The continuos lines are fitted with the form $\sim \exp \left(-b / \sqrt{t_{c}-t}\right)$ with $t_{c}=1$ and $b$ a fitting parameter. The thermodynamic limit is extrapolated by using chains of length up to $2 n=60$. All the DMRG simulation are performed by keeping at most 1024 DMRG states and 5 finite size sweeps with an error energy $<10^{-9}\left(10^{-7}\right)$ for $s=1(s=2)$.

of the entanglement entropy [12]. Very recently, a deformed version of Motzkin [14] and of Fredkin [15] chains have been 
introduced, and their gap studied [16], with the contribution of Motzkin or Fredkin paths to the ground-state being weighted through the introduction of a parameter $t$.

Due to the aforementioned arguments it appears clear as these new models are both very interesting by themselves and they could open the path towards fundamental applications. This motivates us to investigate a Motzkin chain for different spin values and in presence of path deformations. Here after an introduction of the model in terms of deformed Motzkin paths, we present density matrix renormalization group (DMRG) [17] calculations which allow to reveal the appearance of different phases as a function of the deformation parameter $t$. In particular we show that local magnetization is able to capture the $t>1$ regime where a clear domain wall structure takes place independently by the spin value $s$. From the other side once $t<1$ the system undergoes to a phase transition of a Berezinskii-Kosterlitz-Thouless (BKT) type [18] as signaled by an exponential opening of the gap. Moreover our numerical calculations confirm that for this kind of deformation the entanglement entropy is bounded and size independent [14]. Crucially we find that this gapped regime can be described solely by a non-vanishing value of SOP thus showing the topological nature of the $t<1$ deformed Motzkin chains. For $s=1$ only one SOP is finite, similarly to what happens in the $S U(2)$-Haldane phase for XXZ or AKLT spin-1 models, thus revealing the presence of a symmetry-protected topological (SPT) order. On the other hand, for $s=2$ different kinds of Haldane phases have been obtained [19, 20]. In particular, for the spin 2 Motzkin chain, we show that two SOPs display a constant decay exhibiting a phase similar to the $S O(5)$ topological Haldane order occuring in $s=2$ AKLT model [21]. Interestingly, unlike the undeformed case $t=1$, for $t<1$ the CDP [22] is valid.

Model. The spin model we consider has the peculiarity of having a ground state which can be expressed in terms of Motzkin paths describing all the possible $2 n$ moves that one can make to go from a point of height $h=0$ to an other point of the same $h$ without crossing the 0 line [10, 11]. As shown in Fig. 1 $a$ ) and $b$ ), spins can be seen as moves by imposing that up/zero/down spin corresponds to increasing/conserving/decreasing the height of the path. Of course, for spin $s=1$ only uncolored steps (uncolored Motzkin chain) are allowed, while larger values of $s$ can be achieved when colored steps are possible (colored Motzkin chain). The Hamiltonian reads:

$$
H=\sum_{j=1}^{2 n-1} \Pi_{j, j+1}(s, t)+\Pi_{\partial}(s)+\sum_{j=1}^{2 n-1} \Pi_{j, j+1}^{\text {cross }}(s)
$$

where $\Pi_{j, j+1}(s, t)=\sum_{k=1}^{s}\left(\left|\phi(t)^{k}\right\rangle\left\langle\left.\phi(t)^{k}\right|_{j, j+1}+\right.\right.$ $\left|\psi(t)^{k}\right\rangle\left\langle\left.\psi(t)^{k}\right|_{j, j+1}|+| \Theta(t)^{k}\right\rangle\left\langle\left.\Theta(t)^{k}\right|_{j, j+1}\right)$ is the bulk term, $\Pi_{\partial}(s)=\sum_{k=1}^{s}|-k\rangle\left\langle-\left.k\right|_{1}+\mid k\right\rangle\left\langle\left. k\right|_{2 n}\right.$ is the boundary term which makes more favorable for the first spin to point upward, $|k\rangle$, and the last downward, $|-k\rangle$. The latter term in Eq. (1p $\Pi_{j, j+1}^{\text {cross }}(s)=\sum_{k \neq k^{\prime}}=\left|k,-k^{\prime}\right\rangle\left\langle k,-k^{\prime}\right|$, present only for $s>1$, ensures the color matching

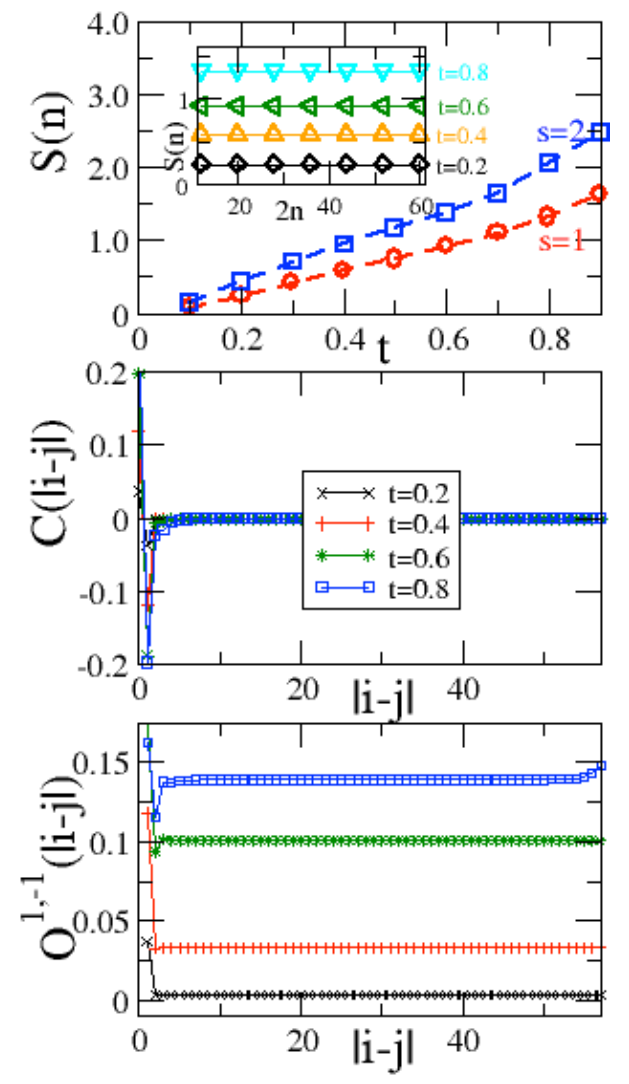

Figure 2: (Color online) a): Entanglement entropy $S(A)$ for a subsystem having length $n$ with $1 \leq i \leq n$ for $s=1$ (red symbols) and $s=2$ (blue symbols). The inset shows the constant behavior of $S(A)$ as a function of the size $2 n . b): C(|i-j|)$ for different $t<1$ values. $c): O^{1,-1}(|i-j|)$ for different $t<1$ values. The correlations in the panels $b$ ) and $c$ ) are evaluated in a system of size $2 n=60$ with $i$ pinned in the first chain site. We checked that different $i$-values do not alter the physical behavior of the correlations.

of up and down spins with the same $h$. The parameter $t$ appearing in $\Pi_{j, j+1}(s, t)$ describes path deformations and $\left|\phi(t)^{k}\right\rangle=\left(1+t^{2}\right)^{-1 / 2}(|k, 0\rangle-t|0, k\rangle)$, $\left|\psi(t)^{k}\right\rangle=\left(1+t^{2}\right)^{-1 / 2}(|0,-k\rangle-t|-k, 0\rangle)$ and $\left|\Theta(t)^{k}\right\rangle=\left(1+t^{2}\right)^{-1 / 2}(|k,-k\rangle-t|0,0\rangle)$. The deformation induced by $t \neq 1$ keeps the model frustration-free [14], and, while for $t=1$ we recover the undeformed model [10-12], for $t>1(t<1)$ the paths having larger (smaller) $h$ are favored in the ground state. Notice that for $t=1$ one can have analytical expressions for the magnetizatazion and the $z-z$ correlation functions, which were tested against DMRG results in [12]. However, for $t \neq 1$ the corresponding results are not available and therefore we will rely on DMRG results to have a physical description of the properties of the model.

$t \geq 1$ Regime. This latter point explains the $t>1$ behavior of the local magnetization $\left\langle S_{j}^{z}\right\rangle$ observed in Fig. 1 $c$ c) and $d$ ) for $s=1$ and $s=2$ respectively. Indeed, since $t>1$ makes more probable higher paths, in terms of spins this corresponds to a domain wall (DW) structure where the up and down spins are separated in two different regions of equal length $n$ [23] 
and the zero spins are basically absent. Relevantly, as shown in Fig. (1 e) and $f)$, this latter regime is gapless $(\Delta=0)$ meaning that the difference between the ground-state $E_{0}$ and the first excited state $E_{1}$ energy goes to zero in thermodynamic limit (TDL). The aforementioned features allow to find the analogy between Eq. 1 and the XXZ chains for both spin 1 and 2 [24] for strong negative $z$-anisotropies. Further similarities can be also noticed for the $t=1$ case where a gapless regime is associated to a power-law decay of the correlation function $\left\langle S_{i}^{+} S_{j}^{-}\right\rangle$and, as exactly shown in [12], an exponential decay of $\left\langle S_{i}^{z} S_{j}^{z}\right\rangle-\left\langle S_{i}^{z}\right\rangle\left\langle S_{j}^{z}\right\rangle$ [25] thus resembling the $X Y$ phase of XXZ models but, with the key feature that both $\mathrm{AL}$ decay and CDP are violated for $s=2$.

$t<1$ Regime. From the other side, as already mentioned, a $t<1$ deformation minimizes the height of the possible paths. This is clearly visible in the $\left\langle S_{j}^{z}\right\rangle$ behavior shown in Fig. 1 c) and $d$ ) where an almost totally flat local magnetization with $h=0$ is observed. Crucially $\left\langle S_{j}^{z}\right\rangle$ shows also antiparallel peaks at the edges of the chain thus supporting the possible presence of edge states. This effect, as explained before, is produced by the $\Pi_{\partial}(s)$ term in Eq. (1) which has also the role of breaking the ground-state degeneracy. The almost flat magnetization can explain also the fact that the entanglement entropy, $S(A)=-\operatorname{Tr} \rho_{A} \log _{2} \rho_{A}$ of a subsystem $A$, is bounded and does not depend on neither the chain nor on the partition length [14], meaning that the AL scaling is fulfilled. Indeed, as it is possible to see in Fig. 2 $a$ ), we find that $S(A)$ is constant at fixed $t$ for any $2 n$ while it grows almost linearly with the deformation strength. The latter is easily explained by the fact that for $t<1$ the strength of $t$ actually affects mainly the first and the last move with flat $\left\langle S_{j}^{z}\right\rangle=0$ in the bulk. Consequently a larger/smaller $t$ will produce a higher/lower value of $\left|\left\langle S_{i}^{z}\right\rangle\right|$ in the first and last site, as shown in Fig. 11. thus generating more/less entropy, which is however size-independent (see the inset in the top panel of Fig. 2) because of the flatness of the paths that mainly contribute to the ground state. Notice that, as shown in Fig. 2 $a$ ), this behavior holds for any considered $s$ value. A less trivial aspect, conjectured in [14], emerges by looking Fig. 11 e) and $f$ ), namely $t<1$ deformations support the presence of finite gap in the TDL. As visible in the same figures, for both $s=1$ and $s=2$ the gap opens compatibly with an exponential decay $\Delta \sim \exp \left(-b / \sqrt{t_{c}-t}\right)$, being $t_{c}=1$ and $b$ a fitting parameter, thus signaling a BKT-like phase transition. In integer spin chains gapped regime can be usually associated to either antiferromagnetic (AF) order described by the two points correlation functions

$$
C(|i-j|)=\left\langle S_{i}^{z} S_{j}^{z}\right\rangle-\left\langle S_{i}^{z}\right\rangle\left\langle S_{j}^{z}\right\rangle
$$

or to Haldane orders described by a SOP

$$
O^{k, \bar{k}}(|i-j|)=\left\langle L_{i}^{k, \bar{k}} e^{\imath \pi \sum_{i \leq \ell<j} L_{\ell}^{k, \bar{k}}} L_{j}^{k, \bar{k}}\right\rangle
$$

where $L^{k, \bar{k}}=|k\rangle\langle k|-|-k\rangle\langle-k|$. Notice that, for $s=1$, $k(\bar{k})$ can be solely equal to $1(-1)$ thus $L_{i}^{1,-1}=S_{i}^{z}$ while for $s=2, k(\bar{k})$ can take the values $1(-1)$ and $2(-2)$ and

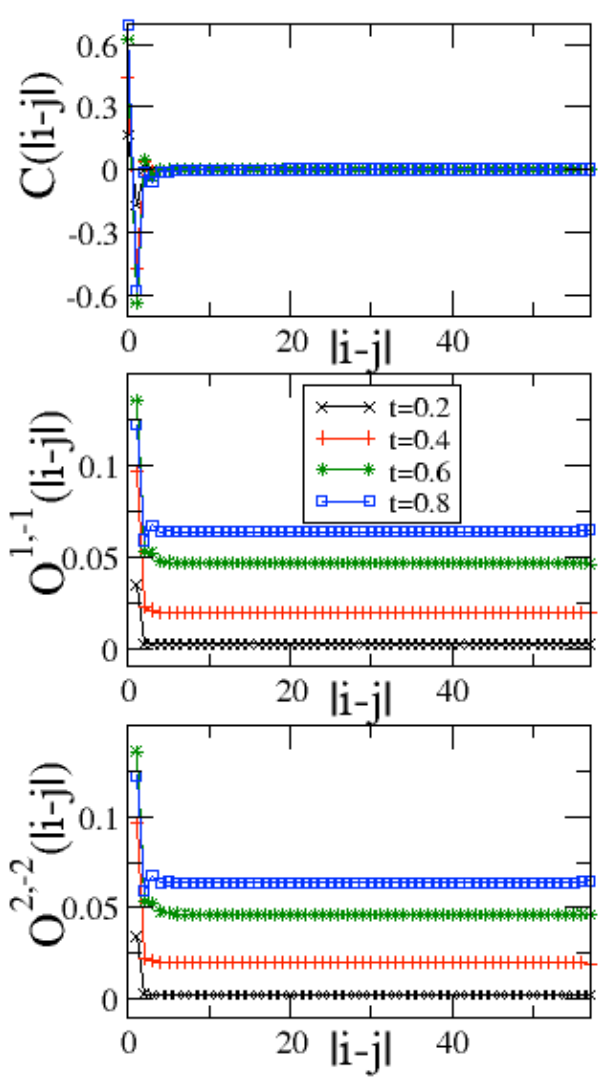

Figure 3: (Color online) $a$ ): $C(|i-j|)$ for different $t<1$ values. $b): O^{1,-1}(|i-j|)$ for different $t<1$ values. $\left.c\right)$ : $O^{2,-2}(|i-j|)$ for different $t<1$ values. All the correlation are evaluated in a system of size $2 n=60$ with $i$ pinned in the first chain site. We checked that using different values of $i$ do not alter the physical behavior of the correlations.

$S_{i}^{z}=2 L_{i}^{2,-2}+L_{i}^{1,-1}$. The important informations encoded in such non-local order parameters is that, once it is finite, Eq. (3) describes a topological phase, usually called HP, with hidden antiferromagnetism (HAF). The HAF order is given by the fact that it can not be described by usual two point correlation functions Eq. (2) thus describing a phase where spins up and down are rigorously alternated and separated by a random number of zero spins. Of course, while for $s=1$ the HP can be given only by alternating +1 and -1 spins thus signaled by a finite $O^{1,-1}(|i-j|)$, for $s=2$ the hidden order can be signaled, as it will be clear in the following, by two or even solely one finite $O^{k, \bar{k}}(|i-j|)$.

$s=1$ case. Here we start our analysis with the $s=1$ case by evaluating both $O^{1,-1}(|i-j|)$ and $C(|i-j|)$ for different $t<1$ values. Fig. 2 clearly shows that while $C(|i-j|)$ rapidly decays to zero the SOP remains constant as a function of the distance thus signaling the presence of a HP. This aspect, in analogy with XXZ chains, supports our prediction regarding the BKT nature of the phase transition. We also checked that the SOPs along transverse directions decay. Furthermore, as visible in Fig. 2 $2 c), O^{1,-1}(|i-j|)$ saturates to a 
constant value which becomes bigger the larger is $t$. At a first look this aspect could seem counterintuitive since one expects that the larger is the gap, the stronger is the SOP. Nevertheless an easy interpretation of the $O^{1,-1}(|i-j|)$ behavior as function of $t$ comes by the geometrical meaning of deformations. Indeed, as explained before, a small $t$ favors the paths with low $h$. Intuitively, one can argue that the path with smaller $h$ is the one where the first and last move corresponds to respectively the rising and the lowering steps with in the middle a series of flat moves. This means that the number of $+1,-1$ spins producing the HAF order is minimized by reducing $t$, thus producing a lower saturation value of the SOP. Nevertheless we checked that even very small deformations support the presence of a constant $O^{1,-1}(|i-j|)$, suddenly disappearing $\left(O^{1,-1}(|i-j|)=0\right)$ for $t=1$, thus allowing to unambiguously conclude that the uncolored $t<1$ Motzkin chain has topological order with HAF. This phase is usually called $S U(2)$-Haldane phase and it has been observed both in spin1 XXZ [6, 26] chains and in the AKLT model [27]. We will keep this nomenclature even if for our model only the operator $\sum_{i} S_{i}^{z}=\sum_{i} L_{i}^{1,-1}$ commutes with the Hamiltonian. This is similar to what happens in the spin-1 XXZ model when a single ion anisotropy term is included, breaking the $S U(2)$ invariance but preserving the HP. For certain systems like the spin-1 XXZ model, the topological order is also captured by an even degeneracy of the entanglement spectrum (ES) [28]. From the other side, in different models, like for instance the AKLT [29] or exotic bosonic Hamiltonians [30], the ES does not present even degeneracy but the topological order is assured by the presence of edge modes and finite SOPs. We checked that this happens also in our case where the ES of $t<1$ deformed Motzkin chains does not display any degeneracy. Nevertheless edge modes, visible in Fig. 1 1 ) and $d$ ), and the finite SOP in Fig 2 assure the topological order [31]. The latter has a further fundamental property due to fact that it appears for an odd value $s$ of the spin. Indeed, once a HP takes place for odd spins $s$, SPT order [7] is generated. This is given by the fact that the edge modes fractionalize in two halfinteger spins which cannot be removed unless in presence of a phase transition or an explicitly symmetries breaking. This consideration allows to conclude that the $s=1$ version of Eq. (1) with $t<1$ deformations supports the presence of SPT topological order with bounded and size independent entanglement thus strongly characterizing the the Motzkin chains.

$s=2$ case. As shown in [11, 12], the $s>1$ undeformed $t=1$ chains have much more intriguing properties with respect to lower spin case. These are induced by the presence of colors which increase the symmetry of the system. As for the $s=1, s=2 \mathrm{XXZ}$ Heisenberg and AKLT models can support the presence of gapped phases for positive $z$-anisotropies. The gap can again be associated to AF order detected by $C(|i-j|)$ or to different kinds of HP, see for instance [19, 32] and reference therein. In particular in such systems SPT topological order is signaled by finite values of both $O^{1,-1}(|i-j|)$ and $O^{2,-2}(|i-j|)$. Moreover, as conjectured in [33] and shown in [32, 34], single ion anisotropy terms can support the formation of a SPT $S U(2)$-Haldane order even for $s=2$. Our calculations in Fig. 1 f) show that again the colored Motzkin chain is gapped for $t<1$ and the gap is associated to HAF since $C(|i-j|)$ has a clear exponential decay rapidly saturating to 0 as shown in Fig. $3 a$ ). From the other side both $O^{1,-1}(|i-j|)$ and $O^{2,-2}(|i-j|)$ have a constant and basically equal behavior thus clarifying that the $s=2$ Motzkin chains with $t<1$ deformations support the presence of an $S O(5)$ HP. It is worth stressing that $S O(5)$ is not the symmetry of our model, rather $U(1) \times U(1) \times \mathbb{Z}_{2}$, since only $\sum_{i} L_{i}^{2,-2}$ and $\sum_{i} L_{i}^{1,-1}$ commute with the Hamiltonian, like in the $\mathrm{s}=2$ AKLT model when the term $\sum_{i}\left(S_{i}^{z}\right)^{2}$ is switched on. Also in that case $S O(5)$-Haldane phase survives once the symmetry is lowered from $S O(5)$ to $U(1) \times U(1)$ [35]. In our case the symmetry is supplemented by the invariace under interchanging the two colors $\left(\mathbb{Z}_{2}\right)$. This is the reason why $O^{1,-1}(|i-j|)$ and $O^{2,-2}(|i-j|)$ are the same, as shown in Fig. 3. Moreover it is important to notice that, in analogy with the $s=1$ case, the SOPs become stronger by increasing $t$. Fig. $3 b$ ) also shows a further information encoded in the $C(|i-j|)$ behavior. Indeed, on the contrary to the $t=1$ regime [12], its exponential decay is associated to a 0 edge-to-edge value thus holding the CDP. The opening of a gap in a colored Motzkin chain, therefore, restores the pure locality of the model in Eq. (1), in agreement with the general findings for gapped local Hamiltonians [36].

Conclusions and Perspectives. In conclusion, our results demonstrate the existence of topological Haldane orders in a new class of spin Hamiltonians. Furthermore we have shown the behavior of Motzkin chains as a function of the deformation strength $t$. While the undeformed $t=1$ case has $X Y$-like features, for $t>1$ the system presents a gapless domain wall structure. From the other side, at $t=1$, we presented evidences of a BKT-like phase transition, characterized by an exponential opening of the gap, occurring for any $t<1$ values. The gapped regime is associated to SPT hidden Haldane antiferromagnetic orders signaled by finite values of string nonlocal order parameters. The two possible Haldane orders have the peculiarity of having an entanglement entropy independent from both block and chain size. Moreover our results suggest that it would be very interesting to have a physical implementation of the Motzkin spin chains. In this respect cold atomic systems, which have been already proposed to simulate several kinds of spin Hamiltonians with topological orders [37], could provide a possible physical platform to implement Motzkin chains. Their experimental realization could be relevant for technological achievements since, from one side, symmetry-protected topological orders have been proposed as ideal candidates towards the realization quantum devices like quantum repeaters [38] and substrate for measurementbased quantum computation [39] while, from the other, side Motzkin paths may have applications in the field of polymer absorption [40]. Finally we underline that, in future works, it would be very interesting to study the gapped regimes in the fermionic version of the $s=3 / 2$ Fredkin model where exotic 
Haldane regimes can take place [41].

Acknowledgments: Discussions with D.K. Campbell and O. Salberger are acknowledged. A. Montorsi and E. Orlandini are warmly are acknowledged for suggesting us crucial references. Useful correspondence with H.-H. Tu is also kindly acknowledged. We acknowledge the support and hospitality of the Simons Center for for Geometry and Physics in Stony Brook where this paper has been conceived during the program Entanglement and Dynamical Systems. LD thanks SISSA and LB thanks University of Padova for kind hospitality. LD acknowledges financial support from MIUR through FIRB Project No. RBFR12NLNA_002. LB and AT acknowledge support from the European STREP MatterWave. LB also acknowledges ERC Starting Grant TopoCold for financial support. Finally, LB and AT acknowledge Karma cluster in Trieste for CPU time.

Note added: After the submission of this paper, two papers appeared on arXiv where a Motzkin spin chain is considered introducing a field theory approach to study certain observables and entanglement measures [42]. We think that addressing with such approach the properties discussed in the present paper would be very interesting, in particular the discussion on the BKT nature of the transition at $t=1$.

[1] A. Auerbach, Interacting electrons and quantum magnetism (New York, Springer-Verlag, 2003).

[2] S. Sachdev, Quantum Phase Transitions (Cambridge, Cambridge University Press, 2011).

[3] X.-G. Wen, Quantum Field Theory of Many-Body Systems (Oxford, Oxford University Press, 2004).

[4] M. A. Nielsen and I. L. Chuang, Quantum computation and quantum information (Cambridge, Cambridge University Press, 2000).

[5] F. D. M. Haldane, Phys. Lett. A 93, 464 (1983); Phys. Rev. Lett. 50, 1153 (1983).

[6] M. den Nijs and K. Rommelse, Phys. Rev. B 40, 4709 (1989).

[7] X. Chen, Z.-Cheng Gu, Z.-Xin Liu, and X.-Gang Wen, Science 338, 1604 (2012); X.-G. Wen, Adv. Phys. 44, 405 (1995); B. Zeng and X.-G. Wen, Phys. Rev. B 91, 125121 (2015).

[8] I. Affleck, T. Kennedy, E. Lieb, and H. Tasaki, Phys. Rev. Lett. 59, 799 (1987); Commun. Math. Phys. 115, 477 (1988).

[9] R. P. Stanley, Enumerative Combinatorics, vol. 2 (Cambridge, Cambridge University Press, 2001).

[10] S. Bravyi, L. Caha, R. Movassagh, D. Nagaj, and P. W. Shor, Phys. Rev. Lett. 109, 207202 (2012); R. Movassagh, J. Math. Phys. 58, 031901 (2017)

[11] R. Movassagh and P. W. Shor, PNAS 113, 13278 (2016).

[12] L. Dell'Anna, O. Salberger, L. Barbiero, A. Trombettoni, and V. Korepin, Phys. Rev. B 94, 155140 (2016).

[13] O. Salberger and V. Korepin, Reviews in Mathematical Physics 19, 1750031 (2017)

[14] Z. Zhang, A. Ahmadain, and I. Klich, PNAS 114, 5142 (2017)

[15] O. Salberger, T. Udagawa, Z. Zhang, H. Katsura, I. Klich, and V. Korepin, J. Stat. Mech: Theory and Exp., 063103 (2017)

[16] R. Movassagh, to appear in Ann. Math. Sci. Appl. 3 (2018) ; L. Levine and R. Movassagh, J. Phys. A: Math. Theor. 50, 255302 (2017)
[17] S. R. White, Phys. Rev. Lett. 69, 2863 (1992).

[18] V. L. Berezinskii, Sov. Phys. JETP 34, 610 (1972); J. M. Kosterlitz and D. J. Thouless, J. Phys. C 6, 1181 (1973).

[19] U. Schollwöck and T. Jolicoeur, Europhys. Lett. 30, 493 (1995); U. Schollwöck, O. Golinelli, and T. Jolicoeur, Phys. Rev. B 54, 4038 (1996).

[20] D. Scalapino, S.-C. Zhang, and W. Hanke, Phys. Rev. B 58, 443 (1998).

[21] H.-H. Tu, G.-M. Zhang, and T. Xiang, Phys. Rev. B 78094404 (2008); J. Phys. A. 41, 415201 (2008); H.-H. Tu, G.-M. Zhang, T. Xiang, Z.-X. Liu, and T.-K. Ng, Phys. Rev. B 80, 014401 (2009).

[22] S. Weinberg, Quantum theory of fields. Foundations (Cambridge, Cambridge University Press, 1995).

[23] The fact the spins up and down are separated in two regions of equal length is due to the fact that we are imposing a vanishing total magnetization $S^{z}=\sum_{j=1}^{2 n} S_{j}^{z}=0$.

[24] V. E. Korepin, N. M. Bogoliubov, and A. G. Izergin, Quantum inverse scattering method and correlation functions ( Cambridge, Cambridge University Press, 1993).

[25] For $s=2$ the exponential decay is verified if the $i$-index in the correlation is sufficiently far from the chain edge, while for $s=1$ the exponential decay is always fulfilled.

[26] T. Kennedy and H. Tasaki, Phys. Rev. B 45, 304 (1992).

[27] T. Kennedy, J. Phys.: Condens. Matter 2, 5737 (1990).

[28] F. Pollmann, E. Berg, A. M. Turner, and M. Oshikawa, Phys. Rev. B 81, 064439 (2010).

[29] H. Fan, V. E. Korepin, and V. Roychowdhury, Phys. Rev. Lett. 93, 227203 (2004).

[30] B. Grémaud and G. G. Batrouni, Phys. Rev. B 95, 165131 (2017)

[31] X. Chen, Z.-C. Gu, and X.-G. Wen, Phys. Rev. B 83, 035107 (2011).

[32] A. Kshetrimayum, H.-H. Tu, and R. Orus, Phys. Rev. B 91, 205118 (2015).

[33] M. Oshikawa, J. Phys.: Condens. Matter 4, 7469 (1992).

[34] H. Aschauer and U. Schollwöck, Phys. Rev. B 58, 359 (1998); K. Nomura and A. Kitazawa, J. Phys. A 31, 7341 (1998); T. Tonegawa, K. Okamoto, H. Nakano, T. Sakai, K. Nomura, and M. Kaburagi, J. Phys. Soc. Jpn. 80, 043001 (2011); Y.-C. Tzeng, Phys. Rev. B 86, 024403 (2012); J. A. Kjäll, M. P. Zaletel, R. S. K. Mong, J. H. Bardarson, and F. Pollmann, Phys. Rev. B 87, 235106 (2013)

[35] H.-H. Tu, R. Orus, Phys. Rev. B 84, 140407(R) (2011)

[36] M. B. Hastings, Phys. Rev. B 69, 104431 (2004); Phys. Rev. Lett. 93, 140402 (2004).

[37] E. G. Dalla Torre, E. Berg, E. Altman, Phys. Rev. Lett. 97, 260401 (2006); M. Dalmonte, M. Di Dio, L. Barbiero, and F. Ortolani, Phys. Rev. B 83, 155110 (2011); K. Kobayashi, M. Okumura, Y. Ota, S. Yamada, and M. Machida, Phys. Rev. Lett. 109, 235302 (2012); I. Cohen and A. Retzker, Phys. Rev. Lett. 112, 040503 (2014); R. M. W. van Bijnen and T. Pohl, Phys. Rev. Lett. 114, 243002 (2015).

[38] F. Verstraete, M. A. Martín-Delgado, and J. I. Cirac, Phys. Rev. Lett. 92, 087201 (2004).

[39] D. V. Else, I. Schwarz, S. D. Bartlett, and A. C. Doherty, Phys. Rev. Lett. 108, 240505 (2012); H. Poulsen Nautrup and T.-C. Wei, Phys. Rev. A 92, 052309 (2015).

[40] E. J. J. van Rensburg, The Statistical Mechanics of Interacting Walks, Polygons, Animals and Vesicles (Oxford, Oxford University Press, 2015).

[41] S. Fazzini, A. Montorsi, M. Roncaglia, and L. Barbiero, arXiv: 1607.05682

[42] X. Chen, E. Fradkin, and W. Witczak-Krempa, 
arXiv:1706.02304; arXiv:1707.02317 